\title{
Estudo de usuários no Arquivo Público do Estado do Maranhão (APEM): analisando as estratégias metacognitivas no processo de busca de informação
}

Dirlene Santos Barros

Professora Assistente da Universidade Federal do Maranhão. Mestre em Ciência da Informação pela Universidade Federal da Paraíba.

Dulce Amélia de Brito Neves

Professora Associada da Universidade Federal da Paraíba. Doutora pela Universidade Federal de Minas Gerais.

A presente pesquisa é fruto da dissertação de Mestrado pela Universidade Federal da Paraíba, que teve como objeto o estudo do processo de busca de informação, desenvolvido pelos usuários do Arquivo Público do Maranhão (APEM). Analisamos, à luz do modelo de comportamento de busca de informação de David Ellis, se as estratégias metacognitivas do profissional da informação do APEM são semelhantes ou diferentes das traçadas pelos pesquisadores no comportamento de busca da informação. Os resultados foram obtidos por meio da aplicação da entrevista semidiretiva, aplicada a dois profissionais, e o protocolo verbal a doze pesquisadores. Destacamos, como resultado, a convergência no comportamento de busca de informação desses sujeitos, com poucas diferenças; e a ausência de um estudo de usuário pelo APEM de forma mais sistêmica e centrada em seus usuários e a criação de uma nova subcategoria.

Palavras-chave: Estudo de usuários; Processo de busca de informação; Arquivo Público do Estado do Maranhão; Metacognição. 


\title{
The State of Maranhão Public Records Customers - A Case Study (APEM):An analysis of the meta cognitive strategies for the information searching process
}

\begin{abstract}
This research is an essay result of Master's degree by Universidade Federal of Paraíba, it had as object of study the process of search of information developed by the users of Public Archive of Maranhão (APEM). We analyzed under the behavior model of search of information by David Ellis, whether the metacognitive strategies of the professional from APEM, whether it looks like or it differentiate from those drawn by the researchers in the behavior of search of information. The results were obtained through the application of semi- directive interview; it was applied to two professionals and the verbal protocol to twelve researchers. We pointed out as result, the convergence in the behavior of search of information of these subjects with few differences; the absence of a user study by APEM in a systemic way and focused on its users and the creation of a new subcategory (copy).
\end{abstract}

Keywords: User studies; Information Search Process; Public Archive of Maranhão; Metacognition.

Recebido em 25.08.2010 Aceito em 12.12.2011

\section{Introdução}

A dinâmica da informação se instaura como objeto de estudo da Ciência da Informação (CI), objeto este que dinamiza, enriquece, gera a sua franca ascensão e a faz despertar para os estudos relacionados ao comportamento humano, frente à informação. Dentre esses estudos, há de se destacar os centrados no usuário (FIGUEREDO, 1994).

Esse panorama vai ao encontro da metamorfose, ora vivenciada pela instituição arquivo, que se direciona para o modelo também centrado no usuário e, não mais no arquivista.

A CI comunga e evoca, assim, uma Arquivologia cada vez mais situada no usuário, tornando-se urgente, sob a ótica teórica e prática, investigações que tenham o usuário como centro do processo arquivístico. 
Diante disso, a presente investigação foi motivada pela busca em compreender a relação entre o estudo de usuários e o arquivo e sua importância para a sociedade da informação. Tivemos como sujeitos os pesquisadores e os profissionais da informação do Arquivo Público do Estado do Maranhão (APEM). Partimos daqui, para analisar as estratégias metacognitivas no processo de busca da informação, a partir do modelo de David Ellis (1989), ampliado por Ellis, Cox e Hall (1993).

Tal panorama teve como objetivo central a análise, à luz do modelo de comportamento de busca de informação de David Ellis (1989), das estratégias metacognitivas do profissional do Arquivo Público do Estado do Maranhão (APEM), a fim de identificar se assemelhavam ou não daquelas desenvolvidas pelos pesquisadores nesse processo.

Sua configuração metodológica está pautada nos relatos orais (protocolo verbal e entrevistas) dos 12 pesquisadores, sendo seis professores universitários e seis historiadores; e dois profissionais do Arquivo, de forma a propiciar uma maior aproximação com o processo de busca de informação e uma análise mais aproximada do seu fazer natural.

Não podemos afirmar que houve uma motivação singular, mas o casamento de vários fatores que nos conduziram à escolha desse estudo. Dentre estes, temos a abordagem teórica e prática desenvolvida durante o cumprimento dos créditos presenciais do mestrado, que possibilitaram visualizar a interação entre o arquivo, o estudo de usuário e a metacognição. Outro fator foi a inexpressividade de pesquisas no campo arquivístico sobre estudos de usuários, a carência do arcabouço teórico, tanto nacional como internacional, sobre esse assunto. Essas evidências foram diagnosticadas por meio de pesquisas feitas em periódicos da área da CI, da Arquivologia, no portal de periódico da Coordenação de Aperfeiçoamento de Pessoal de Nível Superior (CAPES), no portal de Teses e Dissertações do Instituto Brasileiro de Informação em Ciência e Tecnologia (IBICT), no Annual Rewiew of Information Science and Tecnology (ARIST), Journal of Documentation, Proquest, no Journal of the Documentation American Society of Information on Science and Tecnology, dentre outros.

A escolha do APEM, como ambientação do estudo, se configura porque ele custodia o maior acervo documental do Estado. Além disso, em comparação com os demais arquivos da capital do Estado, São Luís, nele é que se pode constatar o maior fluxo de pesquisadores.

Assim, este artigo, em um primeiro momento, traz um caminhar pelas principais concepções teóricas na área de estudo de usuário, arquivo, metacognição e o modelo de Ellis, Cox e Hall (1993). No segundo momento, colocamos em voga os resultados advindos dessa pesquisa, bem como as considerações finais.

\section{0 caminhar teórico como norte para situar o contexto}


O estudo sobre o comportamento informacional do usuário insere-se nos estudos desenvolvidos da CI e de várias outras áreas do conhecimento, que se ocupam da relação do usuário, enquanto ator social, com a dinâmica da informação. Dentre estas áreas, podemos evidenciar a Psicologia Cognitiva, a Comunicação, a Biblioteconomia, a Arquivologia, a Economia, a Antropologia e outras (FIGUEIREDO, 1994).

Esses estudos têm como peça chave o usuário. A concepção em torno de usuário também é complexa. Para Sanz Casado (1994, p. 19), o usuário da informação é "[...] aquel indivíduo que necessita información para el desarrollo de sus actividade [...]", ou seja, é todo indivíduo, pois parte do princípio de que todos nós necessitamos de informação e/ou desejamos informação para desenvolver atividades cotidianas e/ou para saber algo.

Essa necessidade, por sua vez, quando reconhecida pelo usuário, gera a busca pela informação para mudança do seu estado de conhecimento, consistindo, no todo, fazer para satisfazer essa necessidade.

Essa mudança, segundo Wilson (1999), gera o comportamento de busca de informação (information seeking behavior), ou seja, a busca de informação é decorrência de uma necessidade. Em outros termos, trata-se dos métodos utilizados pelo usuário para localizar e acessar as fontes de informação.

É interessante ressaltar que essa abordagem alternativa, ou seja, centrada no usuário, emergiu diversos modelos de estudo de usuário, como o de Tom Wilson (1981; 1996); Brenda Dervin e Nilan (1986); Kuhlthau (1991); e o modelo de David Ellis (1989). Modelo este aperfeiçoado em 1993, pelo próprio Ellis, em conjunto com Cox e Hall (1993).

\subsection{David Ellis: um olhar sobre o processo de busca de informação do usuário}

O modelo de busca de informação de David Ellis parte do pressuposto que o processo de busca se dá por meio de aspectos cognitivos, constituído por características gerais que não são vistas como etapas de um processo (ELLIS, 1989), sendo constituído por oito categorias, a saber:

- iniciar: consiste nas tarefas desenvolvidas no começo da busca, podendo estar presente tanto no início de uma nova atividade como em um novo tópico a ser pesquisado. Para Choo (2003, p. 103), essa tarefa compreende em "[...] identificar as fontes de interesse que podem servir como pontos de partida $[\ldots]^{\prime \prime}$. Essas fontes englobam tanto aquelas bem conhecidas como as menos conhecidas, tendo como condicionante a experiência, o conhecimento prévio ou a inexperiência que o pesquisador tem a cerca da temática a ser pesquisada; 
- encadear: nessa etapa, os pesquisadores fazem relação com a informação encontrada, que, por sua vez, poderá levar a outras citações relevantes. Essa relação poderá ocorrer de duas formas: para trás ou para frente. No primeiro caso, ocorre quando se buscam outras fontes, a partir das referências citadas em um texto específico, isto é, buscam-se as referências ou as fontes citadas no documento consultado; e, no segundo caso, acontece quando "[...] permite localizar material para leitura que cita o texto específico [...]" (CRESPO, 2005, p. 33);

- navegar ${ }^{1}$ : trata-se de uma busca semidirigida em área de interesse do pesquisador. Na realidade, o pesquisador recorre a índices, listas de autores e de títulos, sumários, etc. Segundo Ellis (1989), nessa categoria, o pesquisador passa pelo processo de familiarização com o que é produzido na área, proporcionando-Ihe uma tomada de consciência sobre o que está aparecendo e se encontra disponível na área determinada;

- diferenciar: é a fase na qual o pesquisador "[...] filtra e seleciona as fontes segundo a natureza e a qualidade da informação oferecida [...]" (CHOO, 2003, p. 104), ou seja, é a avaliação que o pesquisador faz em torno das diferentes fontes com objetivo de analisar o material verificado. Dentre os aspectos avaliados estão o teor e a significância da fonte, que têm como finalidade estabelecer uma equiparação entre eles;

- monitorar: consiste em acompanhar a atualização na área de potencial interesse do pesquisador. O foco é determinado pelo próprio pesquisador, com base em sua necessidade. Para González Teruel (2005), o escopo dessa categoria é manter-se atualizado;

- extrair: trata-se de explorar, sistematicamente, uma ou várias fontes, com o objetivo de recuperar materiais de interesse. "[...] Abrange atividades que identificam seletivamente 0 material relevante em uma fonte de informação [...]" (EUCLIDES, 2007, p. 94). É, na realidade, uma atividade direta e focalizada;

- verificar: essa etapa corresponde às atividades em que o pesquisador avalia a validade da informação, a fim de verificar possíveis conformidades ou erros claros; e

- finalizar: está relacionada ao momento em que o pesquisador retorna aos seus escritos para estabelecer ligações entre as suas descobertas e as realizadas por outrem.

\footnotetext{
${ }^{1}$ Para efeito deste estudo será utilizado o termo navegar, apesar de o termo original ser vasculhar. O termo navegar foi adaptado para representar essa categoria em vários estudos (CRESPO, 2005; EUCLIDES, 2007; GARCIA, 2007), devido o contexto informacional que é constituído por uma série de recursos e serviços informacionais.
} 
Ademais, a partir de pesquisas desenvolvidas na base de dados Proquest e no Journal of Documentation, vários outros estudos foram desenvolvidos, aplicando o modelo com as oito categorias. Entretanto, nenhuma dessas investigações foi aplicada a usuários de arquivos, como também não foram analisadas as estratégias metacognitivas.

\subsection{Metacognição: a cognição ativa}

Etimologicamente, metacognição designa o que está para além da cognição (RIBEIRO, 2003), ou seja, é o conhecimento das próprias atividades cognitivas.

Flavell (1979) conceitua a metacognição como qualquer conhecimento ou atividade cognitiva que regula seu objeto qualquer aspecto de uma determinada iniciativa cognitiva, isto é, ela compreende no processo mental, através do qual outras práticas mentais possam ser refletidas.

Para o autor, ela está no plano do monitoramento ativo e do consequente ordenamento e harmonia dos processos mentais relacionados com os dados sobre os quais eles incidem.

Embora o termo metacognição seja recente na literatura, a sua investigação foi principiada no início de 1970, por pedagogos e psicólogos (BROWN, 1987; FLAVELL; WELLMAN, 1977; WEINERT; KLUWE, 1987), que denominaram de metamemória ${ }^{2}$. Por meio deste conceito, eles demonstraram que o processo de estudo e da leitura carregam consigo um tipo de atividade, hoje, conhecida como metacognição.

Ainda para esses autores, o indivíduo tem que aprender quando se deve recorrer a determinadas estratégias (sensibilidade) e fazer o que com o conhecimento sobre a influência das variáveis da pessoa, da tarefa e da estratégia. Esta prática é que torna possível a memorização ou a recordação

variável pessoa - conhecimento geral sobre a própria cognição e sobre a cognição das pessoas. Nessa variável, encontram-se as subcategorias relativas às crenças sobre as diferenças intraindividuais, diferenças interindividuais e regras cognitivas universais;

ii) variável tarefa - conhecimento sobre a natureza das tarefas, o tipo de processamento que deve ser adotado e o gerenciamento necessário para o sucesso no desempenho da tarefa. Um conhecimento metacognitivo dessa subcategoria é o entendimento das implicações da natureza da informação disponível - abundante ou escassa, conhecida ou desconhecida, redundante ou densamente condensada, organizada ou desorganizada, e assim por diante - para

Geralmente, este termo é empregado para representar o conhecimento sobre a memória, mas não contempla o seu controle (STEMBERG, 1979), ou ainda, "[...] um aspecto da metacognição, envolvendo conhecimento e compreensão das capacidades de memória, bem como de meios para aumentar essas capacidades" (STENBERG, 2000, p. 434). 
avaliação da possibilidade de sucesso em atingir os gols pretendidos. Outra subcategoria inclui o conhecimento metacognitivo sobre as demandas e os objetivos das tarefas;

variável estratégia - conhecimento sobre as estratégias prováveis de serem efetivas na obtenção de determinados sub-objetivos e objetivos nos diferentes tipos de empreendimentos cognitivos (NEVES, 2004, p. 33).

O conhecimento metacognitivo, por excelência, traduz o conhecimento da consciência do saber que possuímos sobre as variáveis: pessoa, tarefa e estratégia e a influência sobre o resultado advindo dos processos cognitivos.

Assim, a metacognição é uma ação gerenciada pelo indivíduo, que, ao ter conhecimento dessa habilidade, desenvolve mudanças cognitivas no seu fazer, isto é, ele seleciona, cria, analisa, muda e redireciona sua praxe cognitiva, conduzindo a uma variedade de experiências metacognivas, tanto no que tange a si próprio, como as atividades, as metas e as estratégias.

\section{Metodologia do estudo}

Os sujeitos participantes dessa pesquisa foram 14 pessoas, sendo dois profissionais do APEM, representados por meio das letras A e B; e 12 usuários, sendo seis professores universitários e seis historiadores ${ }^{3}$. A escolha dos profissionais ocorreu em face da experiência no arquivo e da sua atuação no processamento técnico do acervo e/ou no atendimento ao usuário.

Os pesquisadores foram escolhidos pela familiaridade com 0 processo de busca da informação; pelo grau de amadurecimento no que toca a pesquisa e a produção do conhecimento; e pela assiduidade ao APEM.

Esses pesquisadores formaram dois grupos: um instruído sobre o processo de busca de informação, composto por três historiadores e três professores universitários; o outro não instruído, também formado por três historiadores e três professores universitários, aos quais não se fez menção alguma sobre o processo de busca de informação. Esses sujeitos foram representados pela letra PI, para pesquisadores instruídos, e PNI para os não instruídos.

Para os profissionais, foi utilizado a entrevista semidiretiva e para os pesquisadores o protocolo verbal, em que analisamos as próprias categorias do modelo de Ellis, Cox e Hall (1993).

Como instrumento auxiliar às entrevistas e ao protocolo verbal, usamos um conjunto de anotações de campo. Esta ferramenta de apoio se tornou muito útil para registrar nossas impressões sobre alguns detalhes que não haviam sido gravados.

\footnotetext{
${ }^{3}$ Esse quantitativo concerne a realização da investigação após o pré-teste, pois na realização deste, participaram, dois profissionais da informação e dois pesquisadores, sendo um historiador e um professor universitário.
} 


\section{Análise e discussão dos dados}

Convém assinalar que o presente estudo analisou as categorias do modelo de busca de informação de Ellis, Cox e Hall (1993), nas quais buscamos saber:

- iniciar: quais os métodos empreendidos ao começar uma busca de informação?;

- encadear: no processo de busca, é feita alguma conexão entre as referências localizadas?;

- navegar: você faz alguma busca de informação casual na sua área de interesse?;

- diferenciar: a informação obtida passa por alguma filtragem?;

- monitorar: como você se mantém atualizado?;

- extração: é feita alguma seleção de documentos em uma fonte de informação?;

- verificar: as informações recuperadas são checadas?; e

- finalizar: quando o processo de busca de informação termina?

Além disso, buscamos localizar estratégias metacognitivas no desenvolvimento do processo de busca de informação no APEM que não foram pré-definidas, mas foram localizadas na fala dos profissionais e dos pesquisadores.

As estratégias reconhecidas foram as seguintes:

a) agregação de valor (AV): o indivíduo cria conceitos ou atributos que orientam a tomada de decisão em seguir ou escolher um dado caminho;

b) aproximações sucessivas (AS): ocorre quando o indivíduo estabelece passos, em que vai tateando as possibilidades, visando se aproximar, paulatinamente, do objetivo esperado; e

c) compreensão monitorada (CM): acontece quando o sujeito parte do conhecido, no qual visualiza as possibilidades no contexto em que cada passo está sendo dado.

Convém enfatizar, que a pesquisa permitiu com que identificássemos que o estudo de usuário no APEM não é uma ação sistematizada e nem se insere no planejamento do mesmo: "[O estudo de usuário] não é um trabalho sistemático, sempre estamos atentos a buscar essa informação junto ao usuário, indagando sobre suas necessidades de informação, de sua perspectiva de trabalho ou objeto de estudo [...]" (PROFISSIONAL A), ou seja, há o desenvolvimento do estudo de usuário de forma muito tímida e sob a ótica tradicional, com tendência à moderna.

Não querermos dizer com isso que o mapeamento dos pesquisadores que frequentam o Arquivo não seja um ponto fundamental, 
contudo, esse mapeamento deve responder a algumas questões que surgem quando se trata desse tipo de estudo, tais como: qual tipo de informação que esses usuários necessitam? Qual o conhecimento que eles têm da informação? Por que precisam dela? De que forma é feita a seleção das fontes pelos usuários? Por que eles falham na busca da informação? Por que eles não conseguem a informação desejada? Quais os critérios adotados para selecionar a sua informação?

Outra questão evidenciada pelos profissionais e usuários, em relação ao processo de busca de informação no Arquivo, diz respeito aos problemas ou barreiras que atrapalham o desenvolvimento do seu acontecer. Para os profissionais, a maior barreira está alocada no próprio usuário, que não compreende o valor dos conjuntos documentais do Arquivo.

Os usuários creditam a dificuldade no processo de busca, que, segundo eles, ocorre em função da documentação deteriorada pelo tempo e manuseio, além da letra utilizada no período com suas abreviaturas.

No que concerne às categorias do modelo de busca de informação de Ellis, Cox e Hall (1993), todas foram localizadas na pesquisa em questão.

$\mathrm{Na}$ categoria iniciar, todos os participantes tiveram a estratégia Compreensão Monitorada (CM) presente, na qual o conhecimento acerca do Arquivo emerge como um fator determinante na localização, mais precisa e eficaz, dos conjuntos documentais. Outro comportamento em que verificamos o uso dessa estratégia foi a busca de informação em fontes de informação informais. Essa busca, por sua vez, ocorreu com um número mais reduzido dos pesquisadores.

Devemos enfatizar também, que o uso da CM na categoria iniciar corresponde à utilização dos instrumentos de pesquisa pelos sujeitos acima referenciados, é que eles partem do conhecido (uso de instrumentos de pesquisa), no qual visualizam algumas possibilidades sobre as referências identificadas nestes instrumentos, que poderão corresponder ou não a sua necessidade informacional.

Percebemos que, apesar de existir algumas variações quanto a CM entre os profissionais $A$ e $B$, com o grupo dos PI e o dos PNI na categoria iniciar, sob uma olhar holístico, há uma semelhança aproximada no comportamento de busca de informação nessa categoria, ocorrendo em várias etapas do processo de busca de informação, não representando, assim, uma ação estanque, mas, acima de tudo, dinâmica, cuja mobilidade é reflexo das necessidades informacionais emergidas a cada dúvida, questionamento, lacunas nas informações recuperadas, necessitando, assim, algumas vezes ser refeita ou complementada.

No que tange à categoria encadear, percebemos o uso da estratégia aproximação sucessiva (AS), que os profissionais utilizaram o encadeamento para trás (forward chaining). Esse encadeamento foi considerado, nessa pesquisa, importante no processo de busca pelos profissionais, em que as referências e notações de um documento atuam 
como "[...] indicadores para irem à busca de outros documentos [...]." (profissional A).

No caso dos pesquisadores, percebemos as interseções estabelecidas com os dados do documento original com outros em um espaço orientado. Esta situação envolveu a escolha de ir ao encontro ou não da referência sinalizada.

$\mathrm{Na}$ categoria encadear, esperávamos que o uso da estratégia metacognitiva AS pelos profissionais e pelos pesquisadores instruídos e não-instruídos fosse semelhante ou que o grupo dos pesquisadores nãoinstruídos utilizasse em menor proporção tal estratégia.

$\mathrm{Na}$ análise da categoria navegar, a partir das entrevistas e dos protocolos verbais, evidenciamos que os profissionais e os pesquisadores utilizaram, de forma produtiva, a estratégia cognitiva CM, pois ela permite aos mesmos descobrir as possibilidades oferecidas pela documentação.

$\mathrm{Na}$ verdade, essa estratégia é reconhecida, nessa investigação, através da busca nos diferentes tipos de fontes do APEM, mas que mantêm alguma relação com o assunto o qual está sendo pesquisado.

Daí porque essa busca não traz um resultado específico, ou seja, não filtra a informação desejada, mas apresenta os assuntos que, ao primeiro olhar, mantém um relacionamento com a temática específica.

A CM, portanto, apresentou-se como uma estratégia padrão empreendida pelos profissionais pela maior parte dos pesquisadores, tanto instruídos quanto os não instruídos, quando relacionado ao levantamento semi-direcionado feito aos instrumentos de pesquisa.

É interessante frisar que, embora a ação de percorrer a descrição contida nas caixas dos conjuntos documentais, com o intuito de reunir informações sobre a temática a ser pesquisada, tenha utilizado a CM, não figura como padrão de comportamento no processo de busca de informação na categoria navegar, pois somente um pesquisador empreendeu tal ação.

A categoria diferenciar foi marcada pela presença da estratégia Agregação de Valor (AV). O uso da AV estabeleceu uma ligação próxima com os critérios evidenciados na pesquisa de Ellis (1989), Ellis, Cox e Hall (1993), em relação à forma como os cientistas sociais selecionam suas fontes.

No caso específico desta pesquisa, devemos ressaltar que para os pesquisadores, as fontes do APEM, por si só, já são vistas como credíveis. Pode-se, então, perceber que esse posicionamento do pesquisador é permeado pela AV.

De uma forma geral, foi percebido que a $A V$, pelos profissionais $e$ pelos pesquisadores tanto instruídos como os não-instruídos, os conduz ao encontro dos documentos de potencial interesse, como, também, de excluir determinadas fontes que não incidiam sobre esse interesse. 
Não houve, portanto, distanciamentos no uso dessa estratégia nessa categoria. Isto porque as ações empregadas pelos pesquisadores dessa pesquisa foram similares.

A categoria monitorar não foi uma verificada como padrão nesta pesquisa. Isto, porque tal ação foi empreendida, por meio da estratégia CM, apenas por dois participantes: um do grupo dos instruídos (PI4) e outro dos não-instruídos (PNI2).

Percebemos que a ausência dessa categoria no processo de busca de informação dos demais pesquisadores e dos profissionais, deu-se em função do próprio caráter da pesquisa, ou seja, histórica e não queremos dizer com isso que o APEM se configura como um lugar de pesquisa estagnado no tempo, ao contrário, suas fontes documentais, por meio da pesquisa, possibilitam ao pesquisador revisitar teorias existentes, de forma que haja um progresso e uma humanização das mesmas.

Dessa forma, os conjuntos documentais existentes no APEM são sempre atuais e, por consequência, figuram como fontes que possibilitam a atualização da pesquisa e do pesquisador. Por essa razão - apesar de não ter figurado como ação ocorrida no modelo de Ellis (1989) e nem no de Ellis, Cox e Hall (1993) - devemos conceber, como enfatizaram os profissionais, a divulgar dos conjuntos documentais existentes no APEM como uma ação inerente ao monitoramento.

Daí porque sentimos a necessidade de criar uma subcategoria, denominada de divulgar. Esta subcategoria é vista como uma forma de tornar conhecido o que existe no Arquivo. Acreditamos, ainda, que a sua inserção no monitoramento é adequada, em virtude de possibilitar aos pesquisadores a atualização das fontes existentes no APEM, capaz de gerar grandes possibilidades para intervenção e ampliação da pesquisa realizada.

Ao contrário da monitoração, a categoria extrair foi desenvolvida várias vezes por todos os participantes, principalmente por se consubstanciar como busca retrospectiva.

Para tanto, os pesquisadores e profissionais fizeram uso da estratégia metacognitiva AV aos documentos. No caso dos profissionais, a AV foi percebida na consulta direta ao documento e, nos pesquisadores, por meio de duas formas: uma direta e a outra, indireta. A primeira forma é por meio da consulta direta a fonte, conforme desenvolvida pelos profissionais.

O uso dessa estratégia metacognitiva possibilitou tanto aos profissionais quanto aos pesquisadores de ambos os grupos a não recuperarem quantidades elevadas de documentos, pois isso dificultaria, consideravelmente, o processo de análise dos mesmos, que nos direciona a inferir que a AR é uma estratégia metacognitiva presente no processo de busca de informação dos profissionais e dos pesquisadores envolvidos na pesquisa.

A categoria verificar também foi percebida no processo de busca de informação de todos os participantes por meio da estratégia metacognitiva $\mathrm{AV}$, sendo considerada padrão nessa categoria e, por consequência, no 
processo de busca de informação, em que não põem em questão as informações contidas nos documentos. A AV ocorreu em função de possíveis erros quanto à transcrição feita dos originais, sendo envolvida, por uma relação de confiança.

De maneira geral, essa estratégia é desenvolvida de forma discreta, porém tendo algumas semelhanças com a estratégia $A S$, quando os pesquisadores e profissionais buscam conferir as referências indicadas por uma fonte.

Outra categoria presente no estudo foi a finalizar, em que foi identificada a estratégia CM, presente na fala dos dois profissionais. Todavia, essa estratégia não foi reconhecida como padrão do comportamento de busca, em virtude de ter sido identificada em apenas três pesquisadores (PI1, PI4 e PN3).

$\mathrm{Na}$ verdade, a ocorrência dessa estratégia no processo de busca de informação pelos pesquisadores se deu em função de terem terminado a pesquisa que estavam desenvolvendo no momento do protocolo verbal. Isto, ao certo, não simboliza o término de fato, do processo de busca.

A CM só pode ser percebida quando os três pesquisadores reconhecem que o término da pesquisa ocorreu, em virtude do tempo de entrega da pesquisa que já estava expirando.

Além das categorias acima evidenciadas, a pesquisa, em questão, proporcionou o descobrimento de uma nova categoria denominada transcrever. É uma ação própria da pesquisa em arquivos permanentes. Por existirem neles manuscritos, é necessário o conhecimento da Paleografia para que imprima maior fidedignidade possível ao teor do documento.

Essa categoria teve presente, no seu acontecer, a estratégia AV, como condição necessária para que a transcrição não gerasse sentidos contrários, dúbios ou totalmente equivocados com a informação apresentada na documentação. Foi também observada a estratégia AS, por pesquisador (PI5).

$\mathrm{Na}$ realidade, a inserção da transcrição como categoria no modelo de busca de informação de Ellis, Cox e Hall (1993), só é possível aos Arquivos Históricos pela própria peculiaridade da constituição de conjuntos documentais por manuscritos.

\section{Algumas considerações finais}

O acontecer dessa pesquisa possibilitou-nos saber que o caminho percorrido no processo de busca de informação, pelos profissionais e pelos pesquisadores instruídos ou não, não ocorreu de maneira linear e isso foi ao encontro harmônico com o proposto pelo modelo de busca de informação empregado.

Agregado a isso, foi perceptível o grau de semelhança do comportamento de busca dos pesquisadores são similares. Tal postura 
possibilitou verificar a autonomia dos pesquisadores na prática do processo de busca de informação.

Nesta pesquisa, foram percebidas estratégias metacognitivas constantes às categorias do modelo de busca de informação de Ellis (1989); Ellis, Cox e Hall (1993), em que essas estratégias conferiram ao processo uma ação dinâmica, que possibilitaram compreendermos as contribuições dos usuários em âmbito cognitivo

Sobre este contexto, é possível afirmar que a pesquisa respondeu satisfatoriamente aos seus objetivos propostos. Porém, não concebemos esses objetivos como o nosso limite, ao contrário, as suas entrelinhas atuaram como possibilidades a serem desveladas.

Dentre esses desvelamentos, identificamos que o estudo de usuário no APEM, não é uma prática. Isso se deve, a nosso ver, ao fato de que apesar dos profissionais reconhecerem a importância do mesmo, e o abordam a partir da perspectiva tradicional, em que estudam o uso dos conjuntos documentais como forma de satisfação das necessidades informacionais dos seus pesquisadores

Por fim, é possível afirmar, ainda, que a ausência do estudo de usuário no APEM sinaliza, também, a falta de um planejamento que direcione a sua prática, ou seja, é necessário que esse estudo de usuário figure no planejamento do Arquivo como uma ação necessária e constante.

\section{Referências}

BROWN, A. L. Metacognition, executive control, self-regulation, and other more mysterious mechanism. In: WEINERT, F.E.; KLUWE, R. (Org.). Metacognition, motivation, and understanding. Hillsdale,N.J: Erlbaum, 1987. p.1-16.

CHOO, C. W. Como ficamos sabendo: um modelo de uso da informação. In:__ A organização do conhecimento: como as organizações usam a informação para criar significado, construir conhecimento e tomar decisões. São Paulo: Editora SENAC, 2003. cap. 2. p. 63-120.

CRESPO, I. M. Um estudo sobre o comportamento de busca e uso de informação de pesquisadores das áreas de biologia molecular e biotecnlogia:

impactos do periódico científico eletrônico. 2005. 120f. Dissertação (Mestrado em

Comunicação e Informação) - Faculdade de Biblioteconomia e Comunicação,

Universidade Federal do Rio Grande do Sul, Porto Alegre, 2005.

DERVIN, B.; NILAN, M. Information needs and uses. Annual Review of Information 
Science and Techonology. Nova York: Knowledge Industry Publications, v. 21, p. 3-33, 1986.

ELLIS, D. A behavioral approach to information retrieval system design. Journal of Documentation, London, v. 45,n. 3, p. 171-212, sep. 1989.

ELLIS, D.; COX, D.; HALL, K. A comparison of the information seeking patterns of researchers in the physical and social sciences. Journal of Documentation, London, v. 49, n. 4, p.356-369, 1993.

EUCLIDES, M. L. Representação das necessidades de informação na organização da informação: uma análise dos modelos teóricos de busca. 2007. 112f. Dissertação (Mestrado em Ciência da Informação) Faculdade de Filosofia e Ciências, Universidade Estadual Paulista, Marília, 2007.

FLAVELL, J. H. Metacognition and cognitive monitoring:a new área of cognitivedevelopmental inquiry. American Psicoloy, n. 34, v.10, p.906$911,1979$.

FLAVELL, J.H; WELLMAN, H. M. Metamemory. In: KAIL, R.V.; HAGEN, J.W (Orgs.). Perspetives on the development of memory and cognitive. Hillsdale, N.J.: Erlbaum, 1977. p. 3-33.

FIGUEIREDO, N. M. Estudo de uso e usuários da informação. Brasília: IBICT, 1994.

GARCIA, R. M. Modelos de comportamento de busca da informação: contribuição para a organização da informação. 2007. 122f. Dissertação (Mestrado em Ciência da Informação) - Faculdade de Filosofia e Ciências, Universidade Estadual Paulista, Marília, 2007.

GONZÁLEZ TERUEL, A. Los studios de necessidades $y$ usos de la información: fundamentos e perspectivas actuales. Gijón: TREA, 2005.

KUHLTHAU, C. C. Inside the search process: information seeking from the user's perspective. Journal of the American Society for Information Science, Washington, v. 42, p. 361-371, 1991.

NEVES, D. A. B. Aspectos metacognitivos na leitura do indexador. 2004. 130f. Tese (Doutorado em Ciência da Informação) - Escola de Ciência da Informação, Universidade Federal de Minas Gerais, Belo Horizonte, 2004.

RIBEIRO, C. Metacognição: um apoio ao processo de aprendizagem. Psicologia: Reflexão e Crítica, n.16, v.1, p. 109-116, 2003.

SANZ CASADO, E. Manual de estúdios de usuários. Madri: Fundación German Sanches Ruipérez; Madrid: Pirémides, 1994.

STENBERG, R. J. The nature of mental abilities. American Psychologist, v. 34, n. 3, p.214-230, 1979.

Psicologia cognitiva. Porto Alegre: Artmed, 2000. 
WEINERT, F.E; KLUWE, R.H. Metacognition, motivation, and understanding. Hillsdale, N.J.: Erlbaum, 1987.

WILSON, T. D. Exploring models of information behavior: the 'uncertainty' project. Information Processing and Management, Elmsford, NY, v. 35, p. 839-849, 1999.

. Information behavior: an interdisciplinary perspective. London:

The British Library Board, 1996. Disponível em: $<$ http://informationr.net/tdw/publ/infbehav/cont.html $>$. Acesso em: 20 mar. 2008.

. On user studies and information need's. Journal of Documentation, London, v. 37, n. 1, p. 3-15, mar.1981. 